\section{An Iron Nail in the Right Infe- rior Lobe Bronchus in an Adult Retrieved by Bronchoscopic Cryotherapy Technique}

Sir,

An iron nail, as a foreign body, either ingested or aspirated, is uncommonly reported in adults than children. ${ }^{1}$ We present here an unusual case of an adult who inhaled an iron nail, and discuss the method we adopted to remove it.

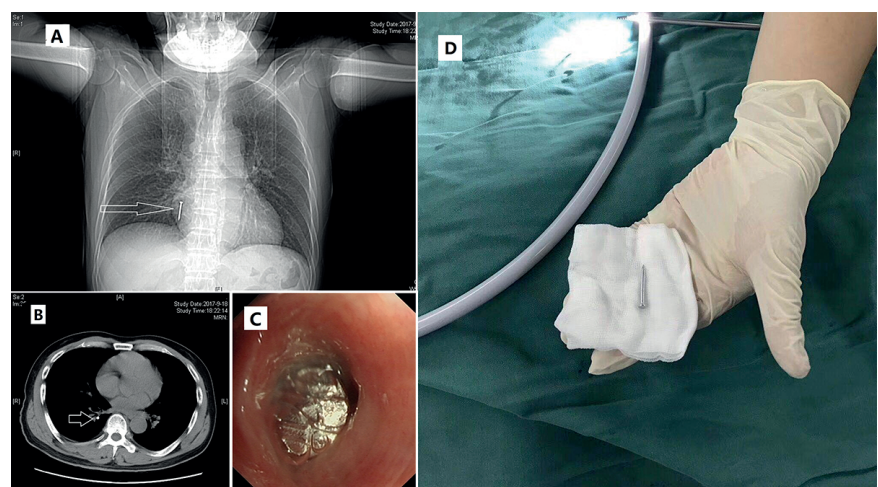

Figure 1: (A) Chest X-ray showing iron nail in right hemithorax. Arrows indicate the iron nail. (B) CT scan of the chest showing iron nail. Arrows indicate the iron nail. (C) The tip of the iron nail as visualized on bronchoscopy. (D) The retrieved iron nail.

A 61-year man presented to the Emergency Department after "having swallowed an iron nail" 26 hours ago. The patient complained of cough and feeling sick but denied any pain, which was consistent with previous reports., ${ }^{2,3}$ Physical examination was normal without any abdominal tenderness, and bowel sounds were normal. A radiography of his chest revealed a nail-shaped foreign body in the right inferior lobe bronchus (Figure $1 \mathrm{a}$, arrow). A computed tomography (CT) scan of the chest further confirmed the chest X-ray findings (Figure $1 \mathrm{~B}$, arrow). A detailed history revealed that he was holding an iron nail in the mouth which was to be nailed in the cement wall. Accidently, he aspirated it in to his lungs. Electronic bronchoscopy revealed that it was an iron nail with a cap (Figure $1 \mathrm{C}$ and D). In this patient, the tip of the nail was facing upwards. An immediate bronchoscopy failed to catch the foreign body. As such, the patient was taken to the operating room and the iron nail was removed with the help of bronchoscopic cryotherapy ${ }^{x}{ }^{4}$ Reinspection by the bronchoscopy showed minor abrasions of the right bronchial mucosa. Later, the patient recovered well and noabnormality on the follow-up examination was found.

It is generally recognised that foreign bodies are prone to lodge into the right bronchial tree. The present case is very similar with previous report in a child. ${ }^{3}$ Children often swallow the metallic objects, but these usually lodge into the bronchial tree due to the negligence of the guardians. Ingestion of iron nail as a foreign body is unusual, especially in adults. Only one case of iron nail inhalation by an adult is recorded, previously. ${ }^{1}$

Our experience from this case has clinical significance, and suggests that bronchoscopic cryotherapy can be used for the removal of foreign bodies that are fragile or difficult to clamp. ${ }^{5}$

\section{CONFLICT OF INTEREST:}

The authors declared no conflict of interest.

\section{AUTHORS' CONTRIBUTION:}

WBY: Data collection, data interpretation and literature search.

GMZ: Study design, data interpretation, manuscript preparation and literature search.

\section{REFERENCES}

1. Panda GP. Iron nail in the Tracheo-bronchial tree. Indian J Otolaryngol 1976; 28(4):192-3.

2. Dutau H, Maldonado F, Laroumagne S, Sophie L. A 'nailbiting' incident!. Respiration 2011; 81:437-8.

3. Deng X, Wang J, Chen R, Huang P, Liu P, Luo X. A straight pin foreign body in a child: Ingested or aspirated? Springerplus 2016; 5:1694.

4. Choe YH, Lee YC, Kim SR. Endobronchial mucormycosis successfully treated with flexible bronchoscopic cryotherapy. Am J Respir Crit Care Med 2018; 198(3):387-9.

5. Dong YC, Zhou GW, Bai C, Huang HD, Sun QY, Huang Y, et al. Removal of tracheobronchial foreign bodies in adults using a flexible bronchoscope: experience with 200 cases in China. Intern Med 2012; 51(18):2515-9.

Wei-Bin Yan ${ }^{1,2}$ and Guo-Ming Zhang ${ }^{3}$

${ }^{1}$ Department of Respiratory, Gaochun Hospital of traditional Chinese Medicine, Nanjing, China

${ }^{2}$ Department of Respiratory, Shuyang People's Hospital, The Affiliated Shuyang Hospital of Xuzhou Medical University, Shuyang, China

${ }^{3}$ Clinical Laboratory, Shuyang People's Hospital, The Affiliated Shuyang Hospital of Xuzhou Medical University, Shuyang, China

Correspondence to: Guo-Ming Zhang, Clinical Laboratory, Shuyang People's Hospital, The Affiliated Shuyang Hospital of Xuzhou Medical University, Shuyang, China

E-mail: gm@xzhmu.edu.cn

Received: July 03, 2019; Revised: September 19, 2019;

Accepted: September 28, 2019

DOI: https://doi.org/10.29271/jcpsp.2020.07.768 